\title{
DROIT DE L'UNION EUROPÉENNE ET DÉMOCRATIE NUMÉRIQUE : CLÉS POUR L'ÉCO-CITOYENNETÉ
}

\author{
JULIEN VIEIRA
}

\begin{abstract}
Afin d'accomplir sa mission fondamentale de levier pour la démocratie, l'Union européenne met à profit les bénéfices des technologies numériques au service de l'information et de la participation environnementale du citoyen. Cette contribution se propose d'aborder les différents outils mis en place par le droit de l'Union européenne pour la mise en œuvre de la démocratie participative environnementale. Cela permettra de mettre en relief la plus-value apportée par ces dispositifs fondés sur l'interopérabilité des systèmes ainsi que l'interactivité entre les différents acteurs qui œuvrent en faveur d'un développement harmonieux et durable pour les générations actuelles et futures.
\end{abstract}




\section{Introduction}

\section{Le système planétaire qu'est devenu Internet s'apparente à un gigantesque système neuro-cérébral semi-artificiel qui combine machines et humains.}

Edgar Morin $(2012,273)$

Les technologies de l'information et de la communication (TIC), et plus particulièrement Internet, sont pensées de telle manière qu'elles constituent un réseau global permettant de relier des ordinateurs connectés sur toute la planète. L'espace de navigation rend possible la liberté d'expression et la liberté de communication sans frontières ainsi que la libre circulation des idées et des informations sans considération de limites territoriales. La langue elle-même n'est plus une barrière dans la mesure où de nombreux sites d'information sont accessibles en plusieurs langues et que certains moteurs de recherche permettent de réaliser une traduction relativement satisfaisante du contenu de ces sites.

Les TIC constituent un vecteur fondamental de la démocratie et plus particulièrement de la démocratie participative. De fait, les technologies numériques ont accéléré le passage inéluctable d'une gouvernance pyramidale à une gouvernance en réseau. Ainsi, on peut constater la mutation du système démocratique moderne hiérarchisé et pyramidal en un nouveau système réticulé, dit post-moderne, qui est horizontal et sans hiérarchie ainsi que le soulignent Gilles Deleuze et Félix Guattari (1980). Le monde juridique prend quotidiennement acte de cet état de fait.

Le droit de l'environnement est sans doute une des branches juridiques qui prend le plus en compte cette transformation démocratique. Effectivement, le principe de participation du citoyen, l'un des principes phares du droit de l'environnement, confirme cette idée de gouvernance en réseau. Il a été le fruit d'un cheminement juridique international dont la consécration la plus emblématique réside dans la Convention d'Aarhus de 1998 sur l'accès à l'information, la participation du public au processus décisionnel et l'accès à la justice en matière d'environnement. En partant $\mathrm{du}$ principe que l'environnement est l'affaire de tous sans aucune considération de frontières, l'engagement du public pour l'environnement s'étend au-delà des limites territoriales. Dans ce cas, les TIC présentent 
donc un moyen supplémentaire de développer une forme d'intelligence collective et cette transparence numérique constitue un nouveau mode d'expression éco-démocratique.

L'Union européenne (UE) a bien compris l'intérêt que représente la démocratie digitale dans la protection de l'environnement. Au sortir de la Seconde Guerre mondiale, l'Europe s'est inscrite dans une vaste entreprise de stabilisation de la démocratie et de la paix entre les nations. C'est donc fidèle à ses principes que l'Union européenne entreprend un aménagement de son ordre juridique pour la mise en œuvre d'une démocratie environnementale effective. Les institutions européennes affichent clairement leur volonté d'inscrire leurs actions dans un cadre plus général de l'Open Government, doctrine de gouvernance qui vise à améliorer l'efficacité et la responsabilité des modes de gouvernance publique par la promotion de la transparence, de la participation citoyenne et d'une collaboration étroite avec les composantes de la société civile (Lathrop et Ruma, 2010).

Par sa règlementation, l'UE constitue un des moteurs de la démocratie environnementale, plus particulièrement en ce qui concerne l'utilisation des TIC. Elle donne, à son niveau ainsi qu'au sein de ses États membres une impulsion toute particulière dans ce domaine ce qui lui permet, en partie, d'atténuer l'idée de déficit démocratique dont elle est taxée plus ou moins injustement (Moravcsik, 2003). Pour accomplir sa mission de consolidation de la démocratie, l'UE donne aux TIC un rôle particulièrement important afin d'assurer le droit à l'information et à la participation du citoyen en matière d'environnement.

\section{L'impact des programmes de l'Union européenne dans l'information environnementale numérique}

Par le biais de différents projets à caractère programmatique, l'UE joue un rôle important dans la diffusion des informations environnementales via les TIC. Les institutions européennes se sont peu à peu rendu compte que les nouvelles technologies pouvaient offrir des possibilités inégalables d'information en temps réel.

Le recours aux TIC permet de prendre le pouls de la planète afin d'assurer l'information environnementale des institutions publiques et des citoyens dans le but d'adopter les décisions les plus opportunes et donc 
d'atténuer les effets du changement climatique. Plusieurs initiatives en cours de réalisation peuvent effectivement être relevées dans ce domaine.

\subsection{Le programme européen de surveillance de la Terre}

Le programme européen de surveillance de la Terre ou Global Monitoring for Environment and Security (GMES) est encadré par le Règlement (UE) n ${ }^{\circ}$ 911/2010 du Parlement européen et du Conseil du 22 septembre 2010 concernant le programme européen de surveillance de la Terre (GMES) et sa mise en œuvre initiale (2011-2013). Cette mise en œuvre de GMES est le fruit d'une collaboration entre l'UE, les États membres et l'Agence spatiale européenne. Le but premier de GMES est "d'offrir, sous le contrôle de l'Union, des services d'information permettant d'accéder à des données et des informations exactes dans le domaine de l'environnement et de la sécurité et conçus pour répondre aux besoins des utilisateurs ${ }^{1} »$. Il s'agit de collecter, et de diffuser ces données. Ce système peut être notamment utile afin de faire face aux catastrophes environnementales naturelles ou dues aux activités humaines (Billet, 2012).

Le règlement $n^{\circ}$ 911/2010 dispose que GMES est conçu pour « être, entre autres, un instrument majeur au service de la biodiversité, de la gestion des écosystèmes, ainsi que de l'atténuation du changement climatique et de l'adaptation à celui-ci ».

Les nouvelles technologies sont au cœur de ce programme et sont utilisées lors des différentes étapes de sa mise en œuvre'. GMES permet, selon l'article 2 du règlement $n^{\circ} 911 / 2010$, la fourniture de services d'accès aux informations environnementales (reposant sur la surveillance de l'atmosphère, la surveillance du changement climatique en appui des politiques d'adaptation et d'atténuation de ses effets, la gestion des urgences, la surveillance des terres, la surveillance du milieu marin et la sécurité) pour les décideurs publics et les particuliers. L'idée est ici de

1. Règlement (UE) no $911 / 2010$ du Parlement européen et du Conseil du 22 septembre 2010 concernant le programme européen de surveillance de la Terre (GMES) et sa mise en œuvre initiale (2011-2013).

2. Surveillance mondiale pour l'environnement et la sécurité (GMES), http://europa.eu/legislation_summaries/other/128170_fr.htm, 29 décembre 2013. 
recourir à un réseau unique qui présente de manière utile toutes ces données par le biais des technologies informatiques.

Dans le but de fournir efficacement ces services d'accès aux informations, l'article $2 \mathrm{du}$ règlement $\mathrm{n}^{\circ} 911 / 2010$ prévoit des observations spatiales de la Terre qui nécessitent l'utilisation des satellites et des observations in situ opérées non seulement par différentes études visant à collecter un grand nombre de données (socio-économiques, géologiques, topographiques, occupation et utilisation des sols, état du sol et biodiversité) mais aussi par l'ensemble des réseaux de sondes mis en place sur terre, en mer, sur les plans d'eau et dans l'atmosphère ${ }^{3}$.

Les différentes composantes de GMES sont coordonnées par la Commission européenne qui est assistée par un comité. Cette coordination s'appuie sur des consultations d'utilisateurs des secteurs public et privé mais aussi sur les comptes rendus $d u$ "forum des utilisateurs» (qui est composé uniquement d'utilisateurs de GMES du secteur public désignés par les États membres) afin de définir au mieux les besoins de ces derniers ${ }^{4}$.

\subsection{Le programme Shared Environmental Information System}

Le cas du programme Shared Environmental Information System (SEIS) constitue également un exemple intéressant d'initiative de l'UE en faveur de l'information environnementale. Depuis quelques années maintenant, l'Union est en train d'élaborer un système communautaire de partage d'informations sur l'environnement. Ce nouvel outil a pour but principal de moderniser et de simplifier la collecte, l'échange et l'utilisation des données et informations nécessaires à l'élaboration et à la mise en œuvre de la politique environnementale. Pour ce faire, l'Union européenne envisage d'intégrer divers instruments de collecte d'informations environnementales dans un réseau décentralisé et électronique déjà existant comme GMES. Il s'agit à la longue de remplacer de manière progressive les systèmes actuels de notification par des systèmes reposant sur l'accès, le partage et l'interopérabilité COM (2008). La motivation principale de la

3. Cf. note 2.

4. Règlement (UE) $\mathrm{n}^{\circ}$ 911/2010 du Parlement européen et du Conseil du 22 septembre 2010 concernant le programme européen de surveillance de la Terre (GMES) et sa mise en œuvre initiale (2011-2013), Article 17. 
Commission européenne figure dans sa communication du 23 janvier 2008 faite au Conseil, au Parlement européen, au Comité économique et social européen et au Comité des régions qui s'intitule "Vers un système de partage d'informations sur l'environnement (SEIS)». La Commission estime que l'ensemble des défis environnementaux actuels comme l'adaptation au changement climatique, l'enrayement de la perte de biodiversité ou encore la gestion des ressources naturelles, nécessitent de «disposer rapidement et facilement des informations liées à l'environnement ». La Commission ajoute que «grâce à la technologie, il est notamment possible à présent de fournir des données en temps réel, ce qui permet de prendre des décisions immédiates et, dans certains cas, de sauver des vies » (COM, 2008).

Cette démarche de simplification repose sur une série de sept principes de manière à rationaliser les procédures de collecte et de diffusion des données environnementales (COM, 2008).

Principes de base du système de partage d'informations sur l'environnement

"Les principes de base du système de partage d'informations sur l'environnement sont les suivants :

- l'information doit être gérée aussi près que possible de sa source ;

- l'information doit être recueillie une seule fois et partagée ensuite entre les différents intéressés pour répondre à des buts multiples ;

- l'information doit être facilement accessible aux autorités publiques et leur permettre de remplir aisément leurs obligations juridiques en matière de notification ;

- l'information doit être facilement accessible aux utilisateurs finaux, essentiellement les autorités publiques à tous les niveaux, du niveau local au niveau européen, afin de leur permettre d'évaluer en temps utile l'état de l'environnement et l'efficacité des actions menées, et d'élaborer une nouvelle politique ;

- l'information doit également être à la disposition des utilisateurs finaux, autorités publiques et citoyens, afin de leur permettre de réaliser des comparaisons à l'échelle géographique appropriée (par exemple, au niveau des pays, des villes, des bassins hydrographiques) et de participer de façon utile à l'élaboration et à la mise en œuvre de la politique environnementale ; 
- l'information doit être totalement disponible pour le grand public, en tenant dûment compte du niveau approprié de compilation et des contraintes de confidentialité, et ce, dans les langues adaptées au niveau national ; et enfin

- le partage et le traitement de l'information doivent être réalisés au moyen d'outils logiciels communs et libres / à source ouverte. » (COM, 2008).

Cette communication du 23 janvier 2008, met également en relief les avantages d'un tel système.

Le premier avantage réside dans la simplification et l'efficacité des procédures. L'institution européenne avance le fait qu'en supprimant les notifications sur papier, les procédures visant à assurer la disponibilité des informations seront plus simples, plus flexibles et plus efficaces.

Le deuxième avantage est celui d'une meilleure réglementation et d'une meilleure politique environnementale grâce à l'amélioration des mécanismes de collecte, d'échange et d'utilisation des données.

Enfin, le troisième et dernier argument repose directement sur la participation du citoyen puisqu'il est question de son implication. En effet, ce dernier argument est pleinement calqué sur la mise en œuvre du principe de participation dans la mesure où il s'agit «d'impliquer les citoyens européens en mettant à leur disposition des informations pertinentes en temps utile, ce qui leur permettra de prendre des décisions relatives à leur environnement en connaissance de cause, et notamment d'agir de façon appropriée en cas d'urgence, et d'influencer les politiques publiques » (COM, 2008).

L'UE mise pleinement sur l'efficacité des TIC de manière à informer le citoyen afin qu'il participe aux décisions ayant un impact direct sur sa sécurité et sa santé. La Commission espère par la même occasion de la part des citoyens « leur réengagement dans le projet européen ».

Malgré l'importance de ces travaux, l'UE ne s'est pas cantonnée à l'élaboration de programmes pour faciliter la diffusion et le partage d'informations environnementales. En effet, il est important pour l'Union que l'ensemble de ces initiatives ne restent pas lettre morte et ne revêtent pas seulement le caractère d'une simple déclaration d'intention au sein des États membres. C'est la raison pour laquelle elle a conféré un caractère contraignant à la diffusion de ces données en élaborant une règlementation précise. 


\section{La directive INSPIRE ou l'encadrement règlementaire de la diffusion des informations environnementales par les TIC}

L'Union européenne joue un rôle important dans l'utilisation des TIC au service de la participation à travers la directive 2007/2/CE du Parlement européen et du Conseil du 14 mars 2007 établissant une infrastructure d'information géographique dans la Communauté européenne du 14 mars 2007, dite INSPIRE (Infrastructure for Spatial Information in Europe) $)^{5}$. Le but de cette directive est de "fixer les règles générales destinées à établir l'infrastructure d'information géographique dans la Communauté européenne, aux fins des politiques environnementales communautaires et des politiques ou des activités de la Communauté susceptibles d'avoir une incidence sur l'environnement ${ }^{6} »$.

Le terme « infrastructure d'information géographique » peut être défini comme un ensemble de services d'information disponibles sur Internet, répartis sur les sites web des différents acteurs concernés, et permettant la diffusion et le partage de données géographiques.

La directive INSPIRE a été élaborée dans le but d'imposer aux autorités publiques de rendre les données géographiques accessibles au public en les publiant sur Internet mais aussi de les partager entre elles. Pour que cette règle européenne soit applicable à une série de données géographiques, cette dernière doit, selon l'article 4 de la directive INSPIRE, obéir à quatre conditions.

Tout d'abord, cette série doit être liée à une zone où un État membre détient et/ou exerce sa compétence.

Ensuite, elle doit se présenter sous forme électronique. En outre, elle doit appartenir à une autorité publique ou à un tiers à la disposition duquel le réseau a été mis ${ }^{7}$.

5. Directive $2007 / 2 / C E$ du Parlement européen et du Conseil du 14 mars 2007 établissant une infrastructure d'information géographique dans la Communauté européenne (INSPIRE), JOUE L 108 du 25/04/2007, p. 1-14.

6. Directive 2007/2/CE du Parlement européen et du Conseil du 14 mars 2007, article $1^{\mathrm{er}}$.

7. Conformément à l'article 12 de la Directive INSPIRE. 
Enfin, il est nécessaire que ces données concernent un ou plusieurs des 34 thèmes définis dans la directive. Effectivement, au sein de chacune des trois annexes de la directive INSPIRE, figure un groupe de thèmes ${ }^{8}$. Ces derniers sont classés dans ces groupes selon leur ordre de priorité, l'annexe 1 citant les thèmes les plus urgents à aborder'.

Lorsque l'on étudie l'intégralité du mécanisme mis en place par la directive INSPIRE, on constate qu'elle présente un certain nombre d'avantages. Cependant, elle contient également des limites qui peuvent clairement réfréner la volonté de démocratisation chère à l'UE.

\subsection{Les avantages de la directive INSPIRE}

Cette directive européenne a été élaborée afin de poursuivre un certain nombre d'objectifs qui contribuent à la protection de l'environnement, enjeu central de ce texte. La réalisation de ces objectifs révèle des avantages non négligeables en termes de gouvernance durable.

8. Les 34 domaines thématiques sont répartis de la manière suivante :

- L'annexe 1 regroupe les données portant sur les référentiels de coordonnées, les systèmes de maillage géographique, les dénominations géographiques, les unités administratives, les adresses, les parcelles cadastrales, les réseaux de transport, l'hydrographie et les sites protégés.

- L'annexe 2 regroupe les données portant sur l'altimétrie, l'occupation des terres, l'ortho-imagerie et la géologie.

- L'annexe 3 regroupe les données portant sur les unités statistiques, les bâtiments, la vocation des sols, la santé et la sécurité des personnes, les services d'utilité publique et services publics, les installations de suivi environnemental, les lieux de production et sites industriels, les installations agricoles et aquacoles, la répartition de la population, les zones de gestion, de restriction ou de réglementation et unités de déclaration, les zones à risque naturel, les conditions atmosphériques, les caractéristiques géographiques météorologiques, les caractéristiques géographiques océanographiques, les régions maritimes, les régions biogéographiques, les habitats et biotopes, la répartition des espèces, les sources d'énergie et les ressources minérales.

9. La directive européenne Inspire: de nouvelles obligations et de nouveaux atouts pour les autorités publiques - Ministère $d u$ Développement durable, http://www.developpement-durable.gouv.fr/La-directive-europeenne-Inspirede.html, 2 janvier 2014. 
Les dispositions de la directive ont d'abord le mérite de s'insérer pleinement dans une logique de démocratie participative. Il s'agit d'élaborer un instrument qui facilite la prise de décision en assurant un niveau raisonnable d'information des autorités publiques, certes, mais aussi de tous les citoyens.

Cette règlementation concourt également à l'ancrage dans les pratiques administratives d'un service d'information du citoyen plus performant et beaucoup plus complet en procédant à un partage de données entre les autorités publiques ${ }^{10}$.

À cela, on peut ajouter que par ces dispositions l'UE encourage l'intelligence territoriale puisque la notion d'interopérabilité est placée au cœur de la directive INSPIRE. L'accent est clairement mis sur l'efficacité puisque cette directive envisage «la possibilité d'une combinaison de séries de données géographiques et d'une interaction des services, sans intervention manuelle répétitive de telle façon que le résultat soit cohérent et la valeur ajoutée des séries et des services de données renforcée ${ }^{11}$ ». La coopération est également le mot d'ordre car la norme européenne insiste sur la mise en commun des données nécessaires à la protection de l'environnement et impose la suppression des entraves à l'échange des informations environnementales entre autorités publiques.

Enfin, on peut également considérer que cette directive met en place un formidable levier pour la croissance économique et l'emploi ${ }^{12}$. De fait, il est indéniable que la création de ces services d'information géographique nécessite et génère un grand nombre d'activités, notamment en ce qui concerne la conception des supports électroniques ou encore la diffusion des données.

\subsection{Les limites de la directive INSPIRE}

La directive INSPIRE, malgré ses nombreux avantages n'est pas exempte de limites. Ces entraves à l'éco-démocratie numérique sont propres au contenu de ce texte règlementaire qui apparaît comme

10. Cf. note 9 .

11. Directive 2007/2/CE du Parlement européen et du Conseil du 14 mars 2007, article 3,7 )

12. Cf. note 9 . 
établissant un dispositif à la carte pour certains des États membres. Néanmoins elles proviennent également de la volonté des pays adhérents et plus particulièrement de la France.

En ce qui concerne les limites intrinsèques à la directive, on peut tout d'abord regretter le fait qu'elle ne concerne que les données numériques. Certaines données sous forme papier peuvent parfois contenir de précieuses informations, ou pire encore, il est tout à fait possible que ces données concernent quelquefois un des 34 thèmes des annexes de la directive. Cependant rien n'oblige les autorités publiques à les numériser.

Rien n'oblige non plus les autorités publiques à compléter l'ensemble des données par des informations qui viendraient à manquer et qui concernent directement l'un de ces 34 domaines thématiques.

Il n'est pas rare que la diffusion de certaines données géographiques s'oppose au droit de propriété intellectuelle. L'article 4-5 de la directive dispose qu'une autorité publique ne peut diffuser les données dont un tiers détient des droits de propriété intellectuelle sans son consentement.

En outre, la directive INSPIRE prévoit une restriction de taille à son application. L'article 4-6 dispose que la directive «s'applique aux séries de données géographiques détenues par une autorité publique ou au nom de celle-ci, lorsqu'elle se situe à l'échelon le plus bas de gouvernement d'un État membre, uniquement si l'État membre a établi des dispositions législatives ou réglementaires qui en imposent la collecte ou la diffusion». Cet article limite considérablement les possibilités de collecter et de diffuser des données géographiques au sein des communes. D'autant plus qu'il arrive fréquemment, qu'au sein d'une commune, des données entrant dans l'un des 34 thèmes de la directive ne puissent être accessibles par voie numérique par absence de disposition législative ou réglementaire. Le plus souvent, les rares données communales bénéficiant de ces dispositions sont les adresses et les documents d'urbanisme ${ }^{13}$. Cependant, ce que l'on peut estimer encore plus critiquable c'est que le pouvoir législatif ou règlementaire de la France ou des autres États membres ne prenne pas ces dispositions afin que l'ensembles des données géographiques importantes figurant dans les annexes de la directive soient collectées et diffusées.

13. Cf. note 9 . 
Enfin, la directive INSPIRE prévoit une dernière limite à son application. Dans son article 13 elle énumère une liste de raisons qui justifient de ne pas diffuser certaines données. Ainsi, la restriction de l'accès au public est justifiée lorsqu'il peut nuire aux aspects suivants : les relations internationales, la sécurité publique, la défense nationale, la confidentialité des travaux des autorités publiques lorsqu'elle est prévue par la loi, la bonne marche de la justice, la confidentialité des informations commerciales ou industrielles, les droits de propriété intellectuelle, la confidentialité des données à caractère personnel, la protection de l'environnement auquel les informations ont trait (par exemple, la localisation d'espèces rares).

L'ensemble de ces limites n'oblige pas les pays à diffuser leurs données dans un certain nombre de cas. Mais, a contrario, rien ne fait obstacle à la diffusion volontaire des dites données par les pays de l'UE. Des pays comme la France pourraient prendre l'initiative, dans les limites de ce qui ne va pas à l'encontre de la sécurité du pays, d'enrichir l'étendue des données mises à disposition. Toutefois il est préférable de ne pas trop en exiger compte tenu que ce même pays a montré des signes de récalcitrance ne serait-ce que vis-à-vis de la simple transposition d'INSPIRE.

Cela nous amène à aborder un second type de limite de la directive qui lui est extérieur. Il concerne la bonne volonté des États membres dans la transposition d'INSPIRE. Cette problématique peut concerner toute directive européenne mais dans notre cas, force est de constater que les pays adhérents n'ont pas tous montré le même enthousiasme dans l'intégration de cette règlementation dans leur ordre juridique interne.

À ce sujet, un focus juridique sur le cas français est particulièrement instructif sur la manière dont les politiques européennes peuvent être court-circuitées par les considérations internes de certaines nations. Cependant, nous pouvons constater que ces résistances ne restent jamais sans conséquence et sont vigoureusement sanctionnées par le juge européen (Huglo et Berger, 2011).

L'ordonnance $\mathrm{n}^{\circ}$ 2010-1232 du 21 octobre 2010 a permis la transposition de la directive INSPIRE de 2007. Elle a ainsi permis de consacrer un chapitre VI intitulé «De l'infrastructure d'information géographique» qui va des articles L.127-1 à L.127-10 du code de l'environnement. Ce chapitre figure dans le Titre II nommé « Information 
et participation du citoyen ». Dans le cadre de cette directive INSPIRE la France a mis en place un Géoportail ${ }^{14}$ permettant d'accéder à l'ensemble des données géographiques (Ledieu, 2006). Mais l'application de la directive INSPIRE par l'État Français ne constitue pas, loin de là, un exploit.

Les problèmes relatifs à la mise en place de l'infrastructure d'information géographique reposent initialement sur la réticence non dissimulée de la France à l'égard de la directive INSPIRE. La France, comme à son habitude, a mis un temps considérable pour transposer une directive européenne (Aubel et Klapisch-Zuber, 2010) ${ }^{15}$. En effet, le délai de transposition de la directive INSPIRE courait jusqu'au 14 mai 2009 alors que l'ordonnance est de 2010. Ce retard a valu à la France une condamnation pour manquement d'État par la Cour de justice de l'Union européenne dans l'affaire Commission européenne/République française du 3 février $2011^{16}$. Bien que la France invoquait devant le juge le fait que la directive serait transposée fin 2010, le juge a rappelé que «l'existence d'un manquement doit être appréciée en fonction de la situation de l'État membre telle qu'elle se présentait au terme du délai fixé dans l'avis motivé et que les changements intervenus par la suite ne sauraient être pris en compte par la Cour» (Picod, 2011).

On peut se demander si cette transposition n'a pas été réalisée uniquement en raison de la condamnation. La raison principale de la réticence de la France réside dans le fait que les budgets permettant le fonctionnement des principaux organismes s'occupant de la gestion du système sont insuffisants pour mener à bien des missions. Ainsi ces organismes comme l'Institut géographique national (IGN) ou encore Météo France commercialisent leur données afin d'obtenir des fonds complémentaires.

14. Le portail des territoires et des citoyens - Géoportail, http://www.geoportail.gouv.fr/accueil, 24 décembre 2013.

15. Il faut savoir que la France n'est pas le seul pays à avoir été montré du doigt en raison de la non-transposition de la directive INSPIRE. Chypre, la Finlande, la Grèce et le Luxembourg ont également subi les foudres de la Commission européenne.

16. CJUE, 3 février 2011, Commission c. République française, aff. C-395/10, Rec. p. I-10*, Pub.somm. 
La directive INSPIRE impose à l'ensemble des pays et donc à l'ensemble des organismes qui ont en charge la mise à jour des portails de mettre à la disposition du public leurs données mais aussi de les partager entre eux. Ce qui veut dire que l'ensemble des données de l'IGN ou de Météo France doivent être libres d'accès, du moins en ce qui concerne les données portant sur les thèmes listés par les 3 annexes de la directive INSPIRE $^{17}$. Cependant le Géoportail mis en place depuis 2006 ne respecte pas toujours les normes de partage de données.

En effet, avec les problèmes de financement des organismes s'entremêlent des problèmes de propriété intellectuelle. Le code de la propriété intellectuelle (CPI) consacre la protection des droits des producteurs de bases de données. Cette consécration a pour conséquence que l'on ne peut utiliser l'œuvre d'un producteur sans son accord. L'article L 342-1 du CPI permet au producteur d'interdire l'extraction et la réutilisation de la totalité ou d'une partie substantielle du contenu de la base $^{18}$. Cependant, l'article L 342-3 du CPI prévoit toute une série de limites qui empêchent l'interdiction par le producteur de l'utilisation de ses données ${ }^{19}$. Notamment l'article L. 342-3, al. $1^{\text {er }}, 1^{\circ}$ dispose que le

17. Il faut savoir que l'IGN comme Météo France ou le BRGM (Bureau de recherches géologiques et minières), dans le cadre plus général de la valorisation des données publiques (Loi du 26 octobre 2005 et ordonnance du 6 juin 2005) peuvent tout à fait licitement diffuser, sous des licences propriétaires des données non listées par la Directive INSPIRE.

18. Article L 342-1 du CPI : «Le producteur de bases de données a le droit d'interdire :

$1^{\circ}$ L'extraction, par transfert permanent ou temporaire de la totalité ou d'une partie qualitativement ou quantitativement substantielle du contenu d'une base de données sur un autre support, par tout moyen et sous toute forme que ce soit ; $2^{\circ}$ La réutilisation, par la mise à la disposition du public de la totalité ou d'une partie qualitativement ou quantitativement substantielle du contenu de la base, quelle qu'en soit la forme.

Ces droits peuvent être transmis ou cédés ou faire l'objet d'une licence.

Le prêt public n'est pas un acte d'extraction ou de réutilisation. ».

19. Article L 342-3 du CPI : (L. no 98-536 du 1er juill. 1998, art. 5) Lorsqu'une base de données est mise à la disposition du public par le titulaire des droits, celuici ne peut interdire : 
producteur ne peut interdire l'extraction ou la réutilisation, par l'utilisateur légitime, de parties non substantielles. C'est ce que l'on appelle le droit de courte citation. Par conséquent, on pourrait penser que les organismes responsables de la diffusion des données dans le Géoportail prévoient la possibilité de télécharger gratuitement les données pour un usage de courte citation. Bien au contraire, tel n'est pas le cas. Les internautes ont la possibilité de consulter gratuitement les données mais lorsqu'il s'agit d'utilisation de ces données, une tarification est prévue au cas par cas selon le type d'usage (Terneyre, 2013).

À l'inverse, les voisins de la France comme l'Espagne ou l'Allemagne figurent parmi les bons élèves (Faucheux et al., 2010). En effet en Espagne, l'Instituto Geográfico Nacional met toutes ses données en ligne en usage libre. L'Allemagne, quant à elle, dispose de nombreux instituts de cartographie dont la subvention est largement assurée par les régions.

Cette situation pourrait donc bien expliquer le retard de la France dans la transposition de la directive INSPIRE. Les organismes chargés de la

$1^{\circ}$ L'extraction ou la réutilisation d'une partie non substantielle, appréciée de façon qualitative ou quantitative, du contenu de la base, par la personne qui y a licitement accès ;

$2^{\circ}$ L'extraction à des fins privées d'une partie qualitativement ou quantitativement substantielle du contenu d'une base de données non électronique sous réserve du respect des droits d'auteur ou des droits voisins sur les œuvres ou éléments incorporés dans la base ;

(L. no 2006-961 du $1^{\text {er }}$ août 2006, art. 3)

$3^{\circ}$ L'extraction et la réutilisation d'une base de données dans les conditions définies aux deux premiers alinéas du $7^{\circ}$ de l'article L. 122-5;

$4^{\circ}$ L'extraction et la réutilisation d'une partie substantielle, appréciée de façon qualitative ou quantitative, du contenu de la base, sous réserve des bases de données conçues à des fins pédagogiques et des bases de données réalisées pour une édition numérique de l'écrit, à des fins exclusives d'illustration dans le cadre de l'enseignement et de la recherche, à l'exclusion de toute activité ludique ou récréative, dès lors que le public auquel cette extraction et cette réutilisation sont destinées est composé majoritairement d'élèves, d'étudiants, d'enseignants ou de chercheurs directement concernés, que la source est indiquée, que l'utilisation de cette extraction et cette réutilisation ne donne lieu à aucune exploitation commerciale et qu'elle est compensée par une rémunération négociée sur une base forfaitaire.

Toute clause contraire au $1^{\circ}$ ci-dessus est nulle. ». 
diffusion des données ne jouent pas le jeu et risqueraient bien d'être contraints, maintenant que la directive est transposée, sous la pression de l'Union européenne, de modifier cette situation. Il reviendrait alors à la France de trouver les fonds nécessaires pour une subvention plus onéreuse de ces établissements publics.

Quoi qu'il en soit, bien que la solution soit imparfaite, la directive INSPIRE constitue pour l'UE un moyen relativement effectif pour assurer la diffusion des informations environnementales sur son territoire par le biais des TIC. Cependant, l'information du citoyen ne constitue pas à elle seule la démocratie participative, elle n'en n'est qu'un des piliers indispensables. La participation du citoyen au processus décisionnel est un élément capital de la nouvelle gouvernance réticulaire qui est en train de se mettre en place. L'UE a compris que les TIC pouvaient faciliter cette mutation. C'est dans ce sens que le Traité de Lisbonne a été élaboré.

\section{Le Traité de Lisbonne générateur d'éco-démocratie numérique}

Il n'est pas rare d'entendre ou de lire que l'UE se caractérise par un réel déficit démocratique (Heuzé, 2005). Ce reproche prend principalement sa source dans le fait que les mécanismes propres aux institutions de l'Union manquent de légitimité et de lisibilité aux yeux des citoyens. C'est la raison pour laquelle il a fallu renforcer cette légitimité et réduire la distance entre gouvernants européens et citoyens. Plus qu'une simple réorganisation de management public, il s'agit d'un réagencement juridique en profondeur.

Effectivement, les Traités de Maastricht, d'Amsterdam et de Nice ont mis l'accent sur la légitimation institutionnelle en élargissant les pouvoirs $\mathrm{du}$ Parlement en termes de contrôle de la Commission et en multipliant les domaines dans lesquels la procédure de codécision doit être appliquée.

Cependant, l'UE n'a pas uniquement renforcé la démocratie représentative. Consciente des mutations des modes de gouvernance, le Traité de Lisbonne permet à l'Union de redorer son blason en misant également sur la démocratie participative.

Certes ce Traité persévère vers la voie de la démocratie représentative en l'affermissant encore un peu plus, toutefois ce texte aménage une place importante à la participation du citoyen au processus décisionnel. Une fois de plus, les TIC vont jouer le rôle de facilitateur démocratique. 
D’une manière générale, il faut savoir que le Traité de Lisbonne a renforcé de façon significative la participation du citoyen au sein de son article $11^{20}$. L'article 11.1 dispose que «les institutions donnent, par les voies appropriées, aux citoyens et aux associations représentatives la possibilité de faire connaitre et d'échanger publiquement leurs opinions dans tous les domaines d'action de l'Union ». Mais la consécration de la participation ne s'arrête pas là puisqu'il est prévu à l'article 11.3 qu'« en vue d'assurer la cohérence et la transparence des actions de l'Union, la Commission européenne procède à de larges consultations des parties concernées ».

Afin de respecter cette disposition du Traité, la Commission européenne a choisi la voie des TIC et s'est doté d'un site internet appelé Your voice qui lui permet d'effectuer des appels à consultation en ligne. Lorsque l'on visite ce site, on peut voir que grand nombre de thèmes environnementaux sont soumis à consultation.

Ainsi, par exemple, les citoyens sont consultés sur des sujets précis comme «les possibilités de révision de la stratégie thématique de l'UE sur la pollution atmosphérique et des politiques connexes", les «options possibles concernant le plan de sauvegarde des eaux européennes » ou encore «les priorités de la politique environnementale de l'UE : Vers un nouveau programme d'action de l'UE en faveur de l'environnement ».

Le site Your voice évoque également la possibilité pour les citoyens de discuter de points importants avec quelques commissaires européens ou de les contacter sur les réseaux sociaux. En ce qui concerne la protection de l'environnement, force est de constater qu'il est ainsi possible de contacter le commissaire au développement sur son blog ou de se rendre sur les comptes Facebook du commissaire de l'environnement ou du commissaire de l'action pour le climat.

De plus, l'article 11.4 du traité de Lisbonne instaure un droit d'initiative citoyenne. L'exercice de ce droit est soumis à plusieurs conditions $^{21}$. En effet, depuis le $1^{\text {er }}$ avril 2012 un groupe d'au moins un

20. Le renforcement de la démocratie européenne, http://europa.eu/legislation summaries/institutional_affairs/treaties/lisbon_treaty/ai0021 fr.htm, 27/12/2012.

21. Petit guide sur l'initiative citoyenne européenne. L'initiative citoyenne en sept étapes, http://www.europarl.europa.eu/news/fr/news-room/content/20120322F CS41704/3/html/L\%27initiative-citoyenne-en-sept-\%C3\%A9tapes, 2 janvier 2014. 
million de citoyens peut inciter la Commission européenne à soumettre une proposition sur un sujet précis. Toujours selon l'article 11.4 du traité de Lisbonne, ces personnes doivent être ressortissantes «d'un nombre significatif d'États membres ». Le règlement n 211/2011 du Parlement européen et du Conseil, du 16 février 2011 relatif à l'initiative citoyenne ${ }^{22}$ précise dans son article 3.2 que « les organisateurs constituent un comité des citoyens composé d'au moins sept membres résidant dans au moins sept États différents ». Les personnes qui entreprennent cette initiative ont un an pour collecter l'ensemble des signatures. Même si le nombre requis de signataires parait important, cette condition peut facilement être atteinte grâce à l'utilisation des nouvelles technologies de manière à créer un réseau suffisamment large pour que les citoyens puissent faire connaitre leur demande et réunir le maximum de personnes.

Justement, l'Union européenne prévoit et facilite cette hypothèse. Effectivement, selon ce même règlement du 16 février 2011, il est prévu que la collecte des signatures se fasse par papier ou par voie électronique. Lorsque les organisateurs à l'origine de l'initiative opèrent le recueil des déclarations en ligne, le système de collecte en ligne doit obéir à des règles spécifiques du point de vue de la protection des données. L'article 6, paragraphe 4 , du règlement relatif à l'initiative citoyenne énonce les «exigences techniques et de sécuritén ${ }^{23}$ » auxquelles le système doit répondre.

22. Règlement (UE) $n{ }^{\circ} 211 / 2011$ du Parlement européen et du Conseil du 16 février 2011 relatif à l'initiative citoyenne, JOUE L 65 du 11/03/2011, p. 1-2.

23. Article $6 \$ 4$ du règlement relatif à l'initiative citoyenne: «Les systèmes de collecte en ligne sont dotés des dispositifs de sécurité et techniques adéquats pour garantir que :

a) seules des personnes physiques peuvent soumettre un formulaire de déclaration de soutien en ligne ;

b) les données fournies en ligne sont collectées et stockées d'une manière sécurisée afin, notamment, de garantir qu'elles ne puissent être ni modifiées ni utilisées à d'autres fins que pour soutenir l'initiative citoyenne concernée et pour protéger les données à caractère personnel d'une destruction fortuite ou illicite, d'une perte fortuite, d'une altération, d'une divulgation ou d'un accès non autorisés ;

c) le système peut générer des déclarations de soutien sous une forme respectant les modèles figurant à l'annexe III, afin de permettre une vérification par les États membres, conformément à l'article 8 , paragraphe 2 . » 
Si ces exigences sont respectées, il est prévu que le système de collecte en ligne soit certifié par les autorités compétentes dans les différents pays de l'Union européenne. Afin de rendre plus facile cette certification, le règlement du 16 février 2011 prévoit qu'un «logiciel libre intégrant les dispositifs de sécurité et techniques adéquats» peut être fourni gratuitement par la Commission européenne aux organisateurs de l'initiative. On peut donc constater que l'Union européenne encourage fortement la gestion de l'initiative citoyenne par la voie électronique pour obtenir un maximum de signatures dans un délai fixé à un an.

De fait, lorsque l'on s'intéresse à l'objet même des initiatives, on s'aperçoit que certaines d'entre elles concernent directement la protection de l'environnement. Par exemple, une initiative intitulée "L'eau et l'assainissement sont un droit humain! L'eau est un bien public, pas une marchandise! » a été enregistrée par un groupe d'organisateurs provenant de 23 pays membres. Cette initiative a fait l'objet d'un site internet ${ }^{24}$ où il est possible pour les internautes d'apporter leur signature pour que l'initiative soient présentée à la Commission puis étudiée par le Parlement européen. Cette collecte a été un franc succès puisqu'elle s'est achevée le 10 septembre 2013 et qu'elle a permis de recueillir 1857605 signatures dont 1547859 en ligne ${ }^{25}$. L'initiative est en cours d'étude par les institutions nationales et européennes.

\section{Conclusion}

Malgré certaines critiques parfois justifiées, l'UE a toujours montré sa détermination à inscrire son action dans un contexte démocratique. En cohérence avec l'interpénétration des ordres juridiques, elle permet progressivement l'intégration du principe de participation en droit de l'environnement au sein des dispositions règlementaires (et peut être un jour fondamentales) européennes. Si cette association sui generis a su prendre en compte la révolution juridique engendrée par l'avènement du post-modernisme, elle a également porté son attention sur un autre type de bouleversement : la révolution numérique.

24. Water is a Human Right, http://www.right2water.eu/, 19 septembre 2013.

25. Merci d'avoir signé!, http://www.right2water.eu/fr/node/434, 19/09/2013. 
Consciente des grandes opportunités que peuvent offrir les TIC dans le droit à l'information et le droit à la participation au processus décisionnel du citoyen, l'UE développe une palette d'instruments capables de mener à bien cet objectif de démocratie environnementale.

Cependant elle n'en est pas moins prudente et lucide vis-à-vis de certains obstacles omniprésents dans la démocratie digitale. La fracture numérique est sans doute l'entrave la plus conséquente dans l'épanouissement de ce type de processus décisionnel. C'est la raison pour laquelle l'Europe a concentré ses efforts sur ce problème afin que les citoyens européens disposent tous d'un accès égal au numérique quelle que soit la région européenne où ils vivent. En effet, à travers les plans d'action eEurope $2002^{26}$ et eEurope $2005^{27}$ ainsi que le programme $22010^{28}$, l'Europe encourage un aménagement numérique harmonieux des territoires au sein de chaque État-membre, mais aussi entre les États membres.

Cependant, étant composée de nations souveraines, l'UE ne peut à elle seule mener à bien sa mission de démocratisation. Ce dessein dépend également de la volonté de l'ensemble des États membres qui doivent mettre tout en œuvre afin de répondre à cette nécessité impérieuse.

\section{Bibliographie}

Aubel C. et Klapisch-Zuber C. (2010). Directive INSPIRE: un nouveau contentieux contre la France. Actu Biodiversité, no 88/89.

Billet P. (2012). Protection communautaire des sols: horizons lointains. Environnement et développement durable, $\mathrm{n}^{\circ} 5$.

26. Communication de la Commission, 13 mars 2001, eEurope 2002 : Impacts et priorités. - Communication en vue du Conseil européen de Stockholm, 23-24 mars 2001 (COM(2001) 140 final) : Non publié au JOCE.

27. Communication de la Commission, 28 mai 2002, au Conseil, au Parlement européen, au Comité économique et social européen et au Comité de régions Plan d'action eEurope 2005 : une société de l'information pour tous (COM(2002) 263 final) : non publié au JOCE.

28. Communication de la Commission, $1^{\text {er }}$ juin 2005, au Conseil, au Parlement européen, au Comité économique et social européen et au Comité des régions intitulé «i2010 - Une société de l'information pour la croissance et l'emploi » (COM(2005) 229 final) : non publié au JOCE. 
COM (2008). Vers un système de partage d'informations sur l'environnement (SEIS), Communication de la Commission au Conseil, au Parlement européen, au Comité économique et social européen et au Comité des régions du 23 janvier 2008, 46 final.

Deleuze G. et Guattari F. (1980). Mille plateaux, Éditions de minuit, Paris.

Faucheux S., Hue C. et Nicolaï, I. (2010). T.I.C. et développement durable: les conditions du succès. De Boeck, Bruxelles.

Heuzé V. (2005). L'Europe désenchantée. JCPG, $\mathrm{n}^{\circ} 30$.

Huglo C. et Berger B. (2011). La nécessaire adaptation de la législation française au droit de l'Union européenne. À propos de la loi du 5 janvier 2011. JCPG, $\mathrm{n}^{\circ} 5$.

Lathrop D. et Ruma L. (dir.) (2010). Open government: collaboration, transparency, and participation in practice, O’Reilly Media, Beijing $\square$; Cambridge [Mass.].

Ledieu M.-A. (2006). Survol cartographique et photographique de la France. Communication Commerce électronique, $\mathrm{n}^{\circ} 9$.

Moravcsik A. (2003). Le mythe du déficit démocratique européen. Raisons politiques, $\mathrm{n}^{\circ} 10$, p. 87-105.

Morin E. (2012). La voie: pour l'avenir de l'humanité, Pluriel, Paris.

Picod F. (2011). Manquement dû à une non-transposition de la directive INSPIRE. $J C P G, \mathrm{n}^{\circ} 7$.

Terneyre P. (2013). Les actifs immatériels des personnes publiques. Revue juridique de l'économie publique, $\mathrm{n}^{\circ} 714$.

La directive européenne Inspire : de nouvelles obligations et de nouveaux atouts pour les autorités publiques - Ministère du Développement durable, http://www.developpement-durable.gouv.fr/La-directive-europeenne-Inspirede.html.

Le portail des territoires et des citoyens - Géoportail, http://www.geoportail.gouv.fr/accueil.

Le renforcement de la démocratie européenne, http://europa.eu/legislation_summaries/institutional_affairs/treaties/lisbon_t reaty/ai0021 fr.htm.

L'initiative citoyenne en sept étapes, http://www.europarl.europa.eu/news/fr/headlines/content/20120329MUN4 2227/html/L\%27initiative-citoyenne-en-sept-\%C3\%A9tapes. 
62 Les cahiers du numérique $-n^{\circ}$ 2/2014

Merci d'avoir signé!, http://www.right2water.eu/fr/node/434.

Surveillance mondiale pour l'environnement et la sécurité(GMES), $\mathrm{http} / / /$ europa.eu/legislation_summaries/other/128170_fr.htm.

Water is a Human Right, http://www.right2water.eu/. 